\title{
What Makes an Act a Pretense One? Young Children's Pretend-Real Judgments and Explanations
}

\author{
Lili $\mathrm{Ma}^{1}$ and Angeline S. Lillard ${ }^{2}$ \\ ${ }^{1}$ Department of Psychology, Ryerson University, 350 Victoria Street, Toronto, ON, Canada M5B 2K3 \\ ${ }^{2}$ Department of Psychology, University of Virginia, P.O. Box 400400, Charlottesville, VA 22904-4400, USA \\ Correspondence should be addressed to Lili Ma; lilima@psych.ryerson.ca
}

Received 16 January 2013; Accepted 19 March 2013

Academic Editor: Tricia Striano

Copyright (c) 2013 L. Ma and A. S. Lillard. This is an open access article distributed under the Creative Commons Attribution License, which permits unrestricted use, distribution, and reproduction in any medium, provided the original work is properly cited.

\begin{abstract}
The present study examined what makes an act a pretense one for adults and preschoolers. Participants watched pretense versus real acts, judged whether each act was pretend or real, and justified their judgment by citing the cues they used. These reported cues are presumed to reflect viewers' conception of what makes an act a pretense one. The results suggested that like adults, 5-yearolds represented pretense behavior in the form of contrasts between pretense and its real counterpart. However, children placed greater weight on deviant content than on behavioral cues, whereas adults used behavioral cues, especially movement, when content information was not available. These results are discussed in terms of how children's intuitive theories of pretense might differ from those of adults.
\end{abstract}

\section{Introduction}

Pretense is one of the earliest symbolic activities of young humans. In pretending, a child projects a mental representation onto reality, in a spirit of fun; the projection is done intentionally and with full awareness, and is often (but not always) accompanied by activities [1]. For example, a boy might take a stick and project onto it his mental representation of a horse, proceeding to "gallop" around with the stick. Children begin to engage in pretend play at around 12 months, and their pretense activities become more elaborate as they move into the central pretend play years of 3 to 5 [2-5]. Adults also engage in various forms of make believe, from pretending a spoon is an airplane in front of their young children to acting on a stage $[6,7]$. Given the prevalence of pretense throughout the lifespan, an intriguing question is how people, especially young children, make pretense interpretations. Pretense involves distortions of the real world. If a young child reads a pretense event literally, his or her developing representations of the real world might get confused [8]. Thus, it is vital that young children interpret pretense in its nonliteral mode and distinguish it from what is real. The goal of this research is to examine how well children, as compared to adults, discriminate certain pretense and real acts and on what basis they think they make their judgments. The findings have the potential to provide insight into how young children's intuitive theories of pretense might differ from those of adults.

Pretense acts differ from real ones in both underlying intentions and external manifestations. Children from ages 3 to 5 are developing an understanding of the former difference, namely, the underlying intentions that are essential in defining pretense versus real behavior (e.g., [9-11]). We focus on the latter difference here: what aspects of external manifestations distinguish pretense acts from real ones, and which of these do adults and children pick up on?

There are at least two ways in which one might use external cues to discriminate pretense and real acts. The first possibility is that the pretenders signal pretending to others verbally. For instance, pretenders might use flagging words like "pretend," "fake," or "not real" when commenting on their own pretense actions. However, such cues are rare: pretending adults seldom explicitly tell young children that they are pretending [12]. The second possibility is that one might decipher pretense based on deviant content and behavioral signs, such as absence of necessary materials or outcomes 
and modifications in the actions of the pretender. This second way might be the most pertinent for very young children's pretense interpretations: deviant content and behavioral signs are the most salient cues to pretense that can be directly observed and easily contrasted with what children already know is real.

In terms of deviant content, pretense acts can differ from real ones in that the necessary materials and outcomes of familiar activities are often absent [4]. In pretense, some necessary materials (were the act real) might manifestly not exist-they might be represented by substitute objects, or might be purely imaginary. For example, when one is pretending to eat, the eating behavior is often performed in the absence of real food. In addition, pretense acts often do not result in the usual outcomes of the activities performed for real $[4,10]$. For instance, when one is pretending to write, it is very likely that there would be no ink traces left on the paper.

Pretense acts can also differ from real ones in terms of the behavioral modifications of the pretender, including aspects of movement and paralinguistic features. As an example of a variation in movements that has been observed in pretense, people move faster when pantomiming pouring into and drinking from a "glass" than they do when executing the same actions with a real bottle and a real glass [13]. In addition, in pretense, movements are often truncated, exaggerated, or oddly timed [12, 14, 15]. With regard to paralinguistic features, both adults and children make sound effects when pretending. For instance, when children are pretending about cars, they sometimes make "vroom vroom" sounds $[6,16]$; when adults are pretending to drink imaginary juice, they often make nonverbal noises that mimic sounds made during the course of real drinking [12]. Changes in voice might also accompany pretense acts, such as mothers talking more loudly and using a more variable pitch when pretending [15].

Thus, pretense activities can involve both deviant content and specific behavioral signs. At issue here is whether these external cues are salient markers of pretense for children. Deviant content, in particular, might readily bring to mind that an act contrasts with the real and thus might lead to a pretense interpretation. For example, upon seeing that there is no real juice in an empty glass, a child might immediately realize that a person who is "drinking" from that glass is pretending. Because pretense is clearly occurring when content is obviously not there, in the present study we did not examine the use of this cue (but see controls in Study 2). However, there are pretense situations in which content information is either absent or insufficient, and it was the cues used in such cases that were of interest here. For instance, a person might pretend to eat but observers might not be able to see whether actual food is involved, or a person might hold a real apple but pretend to bite it in such a way that observers could not see if an actual bite were taken. In such circumstances, children must rely on other cues to tell pretense from real events.

Previous research has shown that pretenders emit nonverbal behavioral cues [12] and observers use such cues to detect pretense. In Richert and Lillard's work [17], adults and children (ages 3 to 10) watched short video clips of mothers engaging in pretense and real snacks and judged whether each event was pretend or real. The clips were selected based on the presence of different behavioral cues that previous research had shown varied with pretense [12], such as mothers looking longer at the child, moving faster, holding their hands at the mouth longer while "eating," and producing pretend sound effects. Content information (e.g., presence or absence of food) was blocked from view by video-editing a small black rectangle over the place where that content would be. In addition, the word "pretend" was not used in the clips. Although there was improvement with age, even the youngest participants made correct judgments most of the time in response to clips containing more marked variation in certain cues, such as the extent to which mothers looked at the child and how rapidly mothers moved. However, in that study it is unclear which cues viewers used to make their judgments, because participants were not asked for the basis of their judgments. When making a pretense interpretation, the viewers could have picked up a different cue in each clip than what the experimenters selected in the stimuli.

The present work addressed this by having adults and preschoolers state what cue they believe they used in making a pretend-real judgment about an action they watched on a film. In this way, it both replicated the prior study by looking at the proficiency at judging real versus pretense acts, and it examined viewers' conception of what makes an act a pretense one. Five-year-olds were tested because they are well-practiced pretenders, nearing the end of the "high season" of pretend play, but they are still developing their ability to judge pretense; not until the age of 7 are children as proficient at judging pretense as adults are [17]. As in Richert and Lillard's work [17], participants watched short video clips of pretense and real acts in which content cues were blocked and judged whether each act was pretend or real ("judgment of the act"); new in this study, they also indicated the cues they believed they had used for that judgment ("reported cues"). We reasoned that the association between the pretend-real judgment and reported cues would reveal viewers' conception of what makes an act a pretense one. First, when asked to explain their pretend-real judgment, we expected that viewers would only report cues that they believe are characteristic of pretense or real behavior. Second, we speculated that viewers might even go so far as to report making their judgments on the basis of cues that were not actually perceptible in the video clips. In such cases, their theories of what should allow them to identify pretense event would actually trump reality, similar to misrecalling sentences or scenes based on typical real-world representations rather than what was actually perceived [18].

To examine what cues viewers are likely to rely on to make pretense or real interpretations, the association between the two categorical variables-judgment of the act and reported cues-was examined by correspondence analysis. Correspondence analysis is an exploratory technique related to principle components analysis. It displays two or more categorical variables in a property space that plots their association in two or more dimensions. The degree of association between two data points can be displayed as 
the distance between them on the plot $[19,20]$. We discuss this method further below.

\section{Study 1}

\subsection{Method}

2.1.1. Participants. Adult participants were 55 college students ( $M=19.5$ years; 28 females). They were recruited from a public university in a small city and received experiment credit for their participation. Child participants were 44 children around 5 years of age $(M=63.3$ months, range $=54.0-71.2$ months; 25 girls). All children were normally developing and had normal vision. Five additional children were excluded due to unwillingness to participate. Children were recruited through a participant database maintained by a research laboratory at a public university or from local preschools (about half from each source). They were predominately from white, middle-class families.

2.1.2. Materials. Twenty video clips of mother-child interactions ( $M=20.1$ seconds, $\mathrm{SD}=0.36$ seconds) were shown to participants. These clips were refined adaptations of those used by Richert and Lillard [17, Experiment 2], who had taken the clips from a study of mother-child interactions [12]. In each clip, a mother engaged in snacking behaviors that were either pretend or real. Half of the clips showed pretense snacking behaviors, and half showed real snacking behaviors.

Each clip had three pertinent characteristics. First, each clip contained at least two instances of complete snacking behaviors (among them eating, drinking, or pouring) that were either pretend or real. Second, the words "pretend" and "real" were not used in any of the clips. Third, effort was made to eliminate obvious signs about the content through video editing using Final Cut Pro: visual signs of content (e.g., the presence or absence of food or drink in the container) were blocked by video-editing a black rectangle over the content, and auditory signs of real content (e.g., the sounds of consuming the food or drink) were removed.

Four adults previewed all the video clips. They were asked to indicate whether they could hear or see any content cues in each clip (e.g., Cheerios being munched, or lack of juice in the cup), without making any pretend-real judgment. All viewers ascertained that one could not see or hear any direct perceptual information indicating the presence or absence of content in the clips.

2.1.3. Procedure. The adult participants viewed the 20 clips in a quiet room. The clips were randomly arranged in two orders. Half of the participants viewed the clips in one order, and half of them viewed the clips in the other order. After viewing each clip, participants judged the veracity of the mother's behavior on a 4-point scale: "real," "probably real," "probably pretend," and "pretend." They also wrote down why they made that judgment ("Please write down the reasons or the cues you have used for your judgment.").

The child participants were tested individually in a laboratory or in a quiet room at their preschools. The procedure was similar to that with the adults, except for the following changes. After each clip, one experimenter asked the child to judge whether the act was pretend or real, by presenting the 4-point scale verbally. Then the child was asked to justify his or her judgment (e.g., "How could you tell that she was really eating/pretending to eat?"). On the response form, a second experimenter noted children's verbal responses and body language if there was any (e.g., child mimicking the mother's exaggerated lip smacking behavior when explaining a pretend judgment). The average number of clips watched was 15 (out of 20; $\mathrm{SD}=4.84$ ). This had no significant impact on the correspondence analysis.

\subsubsection{Data Coding}

Judgment. The total number of judgments made by adults was 1100 and by children was 657. The majority of the children $(70 \%)$ judged every clip as either pretend or real, without including any "probably real" or "probably pretend" responses in their reports. Because of this tendency, and for ease of comparison, data were adjusted so the "probably" judgments were assigned to the definite category. Then each judgment was coded as either correct (PP, a pretense act judged as pretend, or RR, a real act judged as real) or incorrect (PR, a pretense act judged as real, or RP, a real act judged as pretend).

Reported Cues. After excluding unrecognizable reports and "not sure" or "do not know" responses (adults: 72; children: 100), there were 1044 valid reports from adults and 557 from children, and most of these reported cues (adults: 85.7\%; children: 90.2\%) were coded into four categories: movement, content, sound or noise, and general impression, each with two bipolar subcategories indicating either "appropriate" or "variant" features of the observed behavior (e.g., a Content or $v$ Content; see Table 1). For example, "Her chin moved in a way indicating that she was really chewing," "it seemed that there's juice in the cup," "crunching sounds (some participants claimed to use sounds even though they had been edited out, as confirmed by pre-testing)," and "it looked real" were considered appropriate features, meaning consistent with the real behavior. In contrast, "she held her fingers at her mouth for too long and she did not swallow at all," "the cup seemed empty," "couldn't hear any chewing sounds," and "it's just fake" were considered variant or deviant features as compared to those of real behavior.

The remaining reported cues of the adult participants (14.3\%) included references to appropriate or variant features of the mother's coordination of movements (e.g., "talking when should be chewing"), interaction with the child (e.g., "fake interactions with baby"), emotional expressions (e.g., "fake smiles"), and voice (e.g., "she spoke in a much higher pitch"). The remaining reported cues of the children (9.8\%) included references to the mother's desires (e.g., "she's hungry and she wanted to be strong") or intent (e.g., "she wanted to trick the child") (other, see Table 1).

Occasionally a participant noted two different cues for a judgment of a single act. Such instances were coded as two separate observations. Each observation consisted of one 
TABLE 1: Categories of reported cues.

\begin{tabular}{|c|c|c|c|}
\hline Category & Subcategory & Examples & $\begin{array}{l}\text { Variable } \\
\text { name }\end{array}$ \\
\hline \multirow[t]{2}{*}{ Movement } & Appropriate & $\begin{array}{l}\text { She was really } \\
\text { swallowing; the way } \\
\text { her chin moved } \\
\text { indicated real chewing }\end{array}$ & aMovement \\
\hline & Variant & $\begin{array}{l}\text { Exaggerated or too fast } \\
\text { movement }\end{array}$ & vMovement \\
\hline \multirow{2}{*}{ Content } & Present & $\begin{array}{l}\text { Food in the bowl; cup } \\
\text { has weight }\end{array}$ & aContent \\
\hline & Absent & $\begin{array}{l}\text { There's no real food; } \\
\text { cup has no weight }\end{array}$ & vContent \\
\hline \multirow[b]{2}{*}{ Sound/noise } & Appropriate & Snack-related sounds & aSound \\
\hline & Variant & $\begin{array}{l}\text { Pretend or exaggerated } \\
\text { sound effects }\end{array}$ & vSound \\
\hline \multirow{2}{*}{$\begin{array}{l}\text { General } \\
\text { impression }\end{array}$} & Appropriate & $\begin{array}{l}\text { It looked real or } \\
\text { convincing }\end{array}$ & aGeneral \\
\hline & Variant & $\begin{array}{l}\text { It looked pretend or } \\
\text { fake; overacting }\end{array}$ & vGeneral \\
\hline Other & - & $\begin{array}{l}\text { Adult: facial } \\
\text { expressions, voice, } \\
\text { interaction with the } \\
\text { child, and coordination } \\
\text { of movements } \\
\text { Child: desires and } \\
\text { intent }\end{array}$ & - \\
\hline
\end{tabular}

judgment and the corresponding reported cue. For example, if the participant judged a pretense act as pretend and cited both "fake chewing sounds" and "too fast movements," two observations were coded out of this instance: PP with variations in sound/noise ( $v$ Sound) and PP with variations in movement (vMovement).

2.1.5. Reliability. Two trained research assistants coded the reported cues from the response forms; both were blind to the underlying hypotheses. One assistant coded the adult sample, and the other assistant coded the child sample. A third coder coded $40 \%$ of the adult sample and $50 \%$ of the child sample, and she agreed with the first two coders $89 \%$ and $96 \%$ of the time (Cohen's kappa $=.84$ and .95 , resp.). Disagreements were resolved by discussion. All three coders were blind to the pretend-real judgment of the participants when they coded the reported cues.

\subsection{Results and Discussion}

2.2.1. Proficiency at Identifying Pretense versus Real Acts. Preliminary analyses revealed no gender effect on the main variables, so gender was not included in the main analyses. All reported $P$ values are 2-tailed. Proportions of correct judgments of pretense versus real acts were averaged across participants in each age group, and the means and standard deviations are presented in Table 2. Adult participants exhibited a high degree of proficiency. Overall, they correctly judged the clips at an above-chance level ( $88 \%$ correct),
TABLE 2: Mean proportion of correct judgments.

\begin{tabular}{|c|c|c|c|c|c|c|c|}
\hline & \multirow{2}{*}{$N$} & \multicolumn{2}{|c|}{ Pretense acts } & \multicolumn{2}{|c|}{ Real acts } & \multicolumn{2}{|c|}{ Overall } \\
\hline & & $M$ & SD & $M$ & SD & $M$ & $\mathrm{SD}$ \\
\hline \multicolumn{8}{|l|}{ Study 1} \\
\hline Adults & 55 & $.90^{* *}$ & .130 & $.87^{* *}$ & .130 & $.88^{* *}$ & .107 \\
\hline Children & 44 & $.74^{* *}$ & .229 & .46 & .340 & $.61^{* *}$ & .160 \\
\hline \multicolumn{8}{|l|}{ Study 2} \\
\hline Children & 39 & $.83^{* *}$ & .186 & .53 & .332 & $.68^{* *}$ & .147 \\
\hline
\end{tabular}

$t(54)=26.45, P<.001$, which is comparable to the performance of the adults (80\% correct) in Richert and Lillard's work [17, Experiment 2]. More specifically, they correctly identified the pretense acts $90 \%$ of the time and the real acts $87 \%$ of the time, both at above-chance levels, $t(54)=22.56, P<.001$, and $t(54)=21.14, P<.001$, respectively (one-sample $t$ tests).

Children's overall proficiency (61\% correct) was significantly above chance, $t(43)=4.50, P<.001$, which is comparable to that of the preschoolers (57\% correct) in Richert and Lillard's work [17, Experiment 2]. Children correctly identified the pretense acts at an above-chance level (74\% correct), $t(43)=6.97, P<.001$. For the real acts, their performance was at chance ( $46 \%$ correct), $t(43)=-0.74$, $P=.465$. This high error rate is discussed later.

Participants' proficiency was further analyzed using a mixed-design analysis of variance, with clip type (pretense versus real acts) as the within-subjects factor and age (children versus adults) as the between-subjects factor. The results indicated a significant main effect of clip type, $F(1,97)=$ $18.48, P<.001, \eta_{p}^{2}=.160$. In general participants were more proficient at judging the pretense acts $(83 \%$ correct) than the real ones $(69 \%$ correct). The main effect of age was also significant $F(1,97)=117.64, P<.001, \eta_{p}^{2}=.548$, in that adults were more competent than children. The interaction between clip type and age was also significant, $F(1,97)=13.11$, $P<.001, \eta_{p}^{2}=.119$. Paired-samples $t$ tests indicated that adults were equally competent in judging both pretense and real acts, $t(54)=1.21, P=.232$, whereas children were more proficient at judging the pretense acts than the real ones, $t(43)$ $=3.73, P=.001$.

2.2.2. Association between Pretend-Real Judgment and Reported Cues. Correspondence analysis was applied to explore the association between pretend/real judgment and reported cues. This method can represent the association between two or more categorical variables in terms of the distances between the data points on a plot $[19,20]$. In a two-way correspondence analysis, a cross-tabulation table of frequencies is first standardized, so that the relative frequencies across all cells sum to 1.0. The entries in the table of relative frequencies are represented in the form of distances between individual data points of the rows and columns in a low-dimensional space. During this computation, the overall inertia or chi-square for the twoway table is decomposed, where inertia equals the overall 


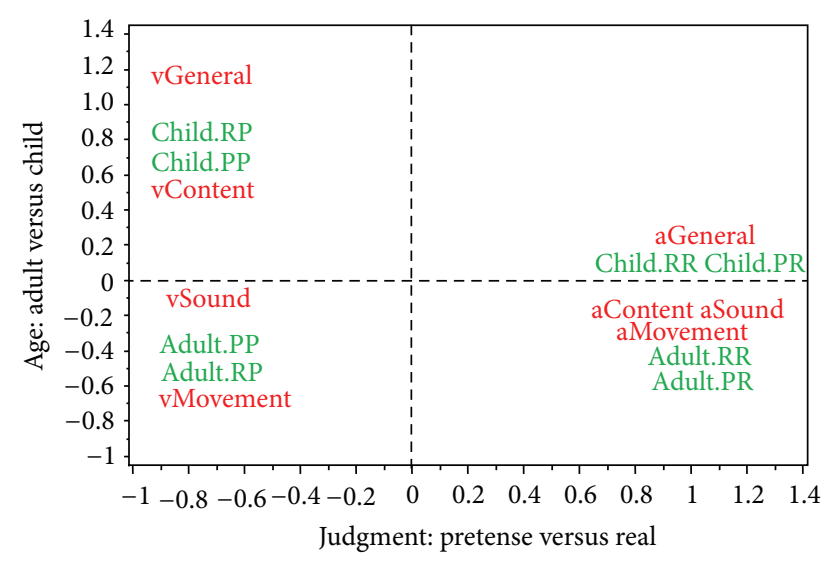

FIGURE 1: Association between judgment and reported cues-adults versus children in Study 1. Judgments identified with "child" were made by children; judgments identified with "adult" were made by adults.

chi-square divided by the total number of observations. On the plot, the distances between the data points represented by the coordinates are weighted (i.e., chi-square) distances between the relative frequencies, which indicate the strength of the association between the data points of the categorical variables (see $[19,20]$, for detailed introduction).

Table 3 shows the frequency of each type of the reported cues, by judgment type and age group. In order to provide a direct comparison between adults and children, data from both age groups were collapsed to carry out a single correspondence analysis, with focus on the four bipolar categories of justifications that represented most of the responses of both ages (e.g., movement, content, sound or noise, and general impression). Age (child versus adult) and judgment type (e.g., PP) were combined as a single, interactive variable (e.g., child.PP and adult.PP). The resulting two-way table was then analyzed, with age-judgment combinations as the rows and reported cues as the columns.

The analysis included 1397 observations (895 adult observations). The results indicated that the first two dimensions could explain $86.9 \%$ of the total inertia (i.e., chi-square) for the association between the participants' pretend-real judgment and the reasons they claimed. Most of the association was in fact attributable to the first dimension, accounting for $69.8 \%$ of the total inertia. A two-dimensional solution therefore has a satisfactory fit to the data and can give an excellent summary of the association between the variables.

Figure 1 shows the association between the participants' judgments and reported cues on those two dimensions. The first dimension, a clear left-right dimension of pretend versus real judgment, shows that cues considered "appropriate" to the activity were closely associated with real judgments, whereas cues believed to be deviant were closely associated with pretend judgments. Normal movements, "expected" normal sounds when consuming the food or drink, and "inferred" existence of content were the most pertinent. On the other hand, an act was judged as pretend when the participant believed that it was accompanied with deviant signs (e.g., "inferred" absence of content, deviant sound effects, or nonstandard movements). Thus, as might be expected, in general both adults and children judged pretense acts by their deviance from their real counterparts.

The second dimension accounts for $17.1 \%$ of the total inertia and displays the age difference. Adults were more likely to cite nonstandard features of movements when justifying their pretense interpretations. In contrast, children tended to justify their pretense interpretations by referring to absence of real content. This is despite the fact that we eliminated content cues by blocking visual information and removing auditory clues like chewing sounds. This is the case for both children's correct detection of pretense (PP) and their false identification of real acts as pretense (RP).

An interesting finding of Study 1 is children's frequent false identification of real acts as pretense (about half the time), seeming to reflect a "pretense bias." In the current stimuli, cues about the necessary content (e.g., food or juice) were blocked. One might worry that this video blocking might have confused children and led to this "pretense bias." This seems unlikely for two reasons. First, pretense clips were edited in the same way, with a black rectangle placed over where the content would be. Second, we know from other research that children can clearly read what is going on behind visual blocks on video-it is the basis for a wealth of research on infant cognition (e.g., [21, 22]). Thus, we speculate that in this study children read the person to be eating (or pretend eating) even when content cues were not visible due to the block.

One possible explanation for the "pretense bias" is that children judge everything they see on television as pretense. Previous research has suggested that before the age of 5 children view what is on television as truly real-when the screen shows an ocean, water will come out if one turns the TV set over [23]. But between the ages of 5 and 12, although they judge actors on television to be "real" people (e.g., Mr. and Mrs. Cosby really are married), they see the acting as "pretend" [24]. It is possible that some children in the current study viewed the mothers in the video clips as acting and thus judged all their behaviors to be "pretend" simply because they were on television. To test this, in Study 2 real content was shown for some clips. If all video material is pretend at this age, then even when real content is available, children should judge the acts as pretense.

A more interesting possibility is that the "pretense bias" reveals something about the basis by which children make pretend judgments. Content cues were blocked in the current stimuli, and in this situation adults easily moved to using behavioral cues, particularly how people moved. For example, on the real clips they used cues to reality such as well-timed or nonexaggerated movement. Five-year-olds, however, did not benefit from such behavioral cues. When content cues were not available, on the real clips, half the time they guessed that the mothers were pretending to eat, and, half the time, they guessed them to be really eating. Why were children not also thrown to guessing by the pretense clips? Perhaps it is because behavioral cues to pretense are marked - when judging the pretense clips, children could and did sometimes use pretender (behavioral) cues. In the real 
TABLE 3: Frequencies of reported cues (number of total observations in parentheses).

\begin{tabular}{|c|c|c|c|c|c|c|c|c|c|}
\hline & \multirow{2}{*}{ Judgment } & \multicolumn{8}{|c|}{ Reported cues } \\
\hline & & aMovement & aContent & aSound & aGeneral & vMovement & vContent & vSound & vGeneral \\
\hline \multirow{4}{*}{ Study 1: adults (895) } & $\mathrm{PP}$ & 0 & 3 & 0 & 0 & 261 & 86 & 92 & 7 \\
\hline & $\mathrm{PR}$ & 17 & 7 & 3 & 3 & 2 & 0 & 0 & 0 \\
\hline & $\mathrm{RP}$ & 1 & 0 & 0 & 0 & 28 & 9 & 5 & 1 \\
\hline & $\mathrm{RR}$ & 282 & 39 & 26 & 14 & 5 & 1 & 2 & 1 \\
\hline \multirow{4}{*}{ Study 1: children (502) } & $\mathrm{PP}$ & 0 & 0 & 0 & 1 & 37 & 102 & 41 & 31 \\
\hline & $\mathrm{PR}$ & 16 & 21 & 10 & 13 & 0 & 0 & 0 & 0 \\
\hline & $\mathrm{RP}$ & 0 & 0 & 0 & 1 & 22 & 77 & 20 & 19 \\
\hline & $\mathrm{RR}$ & 37 & 24 & 9 & 18 & 1 & 1 & 0 & 1 \\
\hline \multirow{4}{*}{ Study 2: children (617) } & $\mathrm{PP}$ & 1 & 0 & 1 & 0 & 68 & 105 & 73 & 25 \\
\hline & PR & 20 & 13 & 8 & 7 & 0 & 0 & 0 & 0 \\
\hline & $\mathrm{RP}$ & 2 & 0 & 1 & 0 & 19 & 64 & 32 & 10 \\
\hline & $\mathrm{RR}$ & 95 & 42 & 15 & 16 & 0 & 0 & 0 & 0 \\
\hline
\end{tabular}

PP: a pretense act judged as pretend; PR: a pretense act judged as real; RP: a real act judged as pretend; RR: a real act judged as real.

clips, however, behavioral cues to reality were not salient to children; lacking access to content cues, they (unlike adults) had to guess. Study 1 strongly suggests this possibility, and Study 2 was undertaken to test it.

One concern that must be addressed is whether our procedure obscured children's ability by asking children to quantify their confidence in their judgment. Past research has suggested that 3- to 5-year-olds show little comprehension of the differences between adverbs that denote different degrees of likelihood of judgment, such as possibly, probably, and definitely [25]. In a related vein, preschoolers have difficulty with multiple possibilities when asked to predict the outcome of undetermined events [26]. Perhaps children in the current study had difficulty processing or considering four possible judgments at the same time, so in Study 2 we simply asked for pretend versus real judgments.

\section{Study 2}

\subsection{Method}

3.1.1. Participants. The final sample included 39 children around 5 years of age $(M=64.9$ months, range $=56.9-73.4$ months; 20 girls). All children were normally developing and had normal vision. Four additional children were excluded due to unwillingness to participate. Children were recruited through a participant database maintained at a public university and predominantly from white, middle-class families.

3.1.2. Materials. The stimuli were 20 video clips, including 16 test clips randomly selected from the ones used in Study 1 ( 8 pretense acts and 8 real acts) and four control clips. Two control clips showed obvious real snacking behaviors, with the content cues visible, so viewers could see the real food or drink. The other two control clips showed obvious pretense acts: in addition to salient behavioral signs, the viewers could clearly see that all the containers were empty.
3.1.3. Procedure. Each child was tested individually in a laboratory room. The experimenter showed the child the 20 video clips, one at a time. At the end of each clip, the child was asked to judge whether the act was pretend or real (e.g., "was she really eating or was she just pretending?") and to justify that judgment (e.g., "how could you tell that she was really eating/pretending to eat?"). A video camera recorded the child's responses. All children watched all four control clips. The average number of test clips watched was 15 (out of $16 ; \mathrm{SD}=2.08$ ).

3.1.4. Coding and Reliability. Children's reported cues were transcribed from videotapes. Two trained research assistants coded the reported cues from the transcripts into the same categories as those in Study 1, and on another occasion they coded children's pretend-real judgments from the videotapes. The coders were blind to the pretend-real judgments of the children when they coded the reported cues. Intercoder reliability based on $40 \%$ of the sample was very good. The coders agreed $99 \%$ of the time on pretend-real judgment (Cohen's kappa $=.98$ ) and $90 \%$ of the time on reported cues (Cohen's kappa $=.88$ ). Disagreements were resolved by discussion.

3.2. Results and Discussion. To preview, the 5-year-olds in this study had no difficulty identifying the control clips (about $90 \%$ correct). Their performance in judging the test clips exhibited patterns similar to those of the 5-year-olds in Study 1. Child data from both studies were collapsed to carry out a single correspondence analysis, which indicated similar results across studies.

3.2.1. Proficiency at Identifying Pretense versus Real Acts. Children correctly detected the "pretend" control clips $86 \%$ of the time, and for $84 \%$ of the correctly identified clips they referred to the absence of real content to justify their judgments. Moreover, children correctly identified the "real" 
control clips $94 \%$ of the time, and they referred to the presence of real food or drink when explaining their judgments (88\%). An examination of individual performance patterns suggests that this proficiency is characteristic of most children: $90 \%$ of the children (35/39) correctly identified at least 3 of the 4 control clips, with $74 \%$ of the children (29/39) correctly identifying all four clips. In other words, 5-yearolds had no difficulty identifying acts displayed on television when it was abundantly clear whether an act was pretend or real. Therefore, the "pretense bias" in Study 1 was not due to children's difficulty in recognizing that events in videos are sometimes "real."

Children's performance in judging the test clips exhibited patterns similar to those of the 5-year-olds in Study 1 (see Table 2). Their overall proficiency $(68 \%$ correct) was significantly above chance, $t(38)=7.28, P<.001$. More specifically, they correctly identified the pretense acts at an above-chance level $(83 \%$ correct), $t(38)=11.19, P<.001$. For the real acts, their performance (53\% correct) did not differ significantly from chance, $t(38)=0.50, P=.619$. Additional analyses revealed that children outperformed the 5 -year-olds in Study 1 (74\% correct) in judging the pretense acts, $t(81)=2.18, P=.032$. Their performance in judging the real acts was comparable to that of the 5-year-olds in Study 1 (46\% correct), $t(34)=0.87, P=.387$.

Thus, reducing the number of judgment choices to two did not significantly reduce children's errors in judging the real acts, suggesting that children's "pretense bias" in Study 1 was not due to difficulty with the 4-point judgment scale. Instead, it seems that false identification of the real acts stemmed from children relying heavily on content to the exclusion of behavioral cues. When content cues were not available, children guessed (50-50) as to whether the real content was behind the video block and made their pretendreal judgment accordingly.

3.2.2. Association between Pretend-Real Judgment and Reported Cues. Table 3 shows the frequency of each type of justifications cited by the children. A correspondence analysis was carried out with the collapsed data of the children from both studies, using the same four bipolar categories of justifications as in Study 1 (i.e., movement, content, sound or noise, and general impression). Study (1 versus 2) and judgment type (e.g., PP) were combined as a single variable (e.g., 1.PP and 2.PP), and the resulting two-way table was then analyzed, with study-judgment combinations as the rows and reported cues as the columns.

The analysis included 1119 observations (617 from Study 2). The results indicated that the first dimension could explain $91.2 \%$ of the total inertia for the association between children's judgment of the acts and the reasons they claimed. The second dimension only accounted for $5.0 \%$ of the total inertia. Thus, a one-dimensional solution has a sufficient fit to the data.

As Figure 2 shows, the first dimension is a clear leftright dimension of pretend versus real judgment, for children from both studies. Along this dimension, cues considered "appropriate" to the activity were closely associated with real

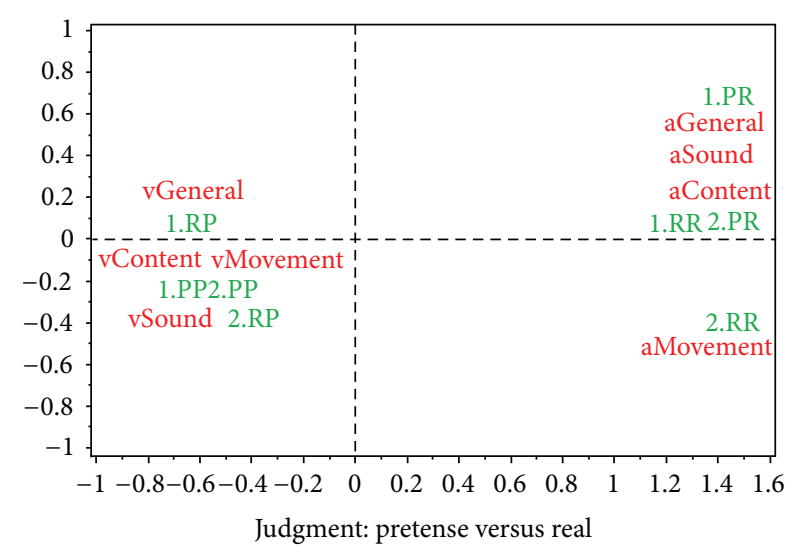

Figure 2: Association between judgment and reported cueschildren in Studies 1 versus 2. Judgments identified with "1" were made by children in Study 1; judgments identified with "2" were made by children in Study 2.

judgments whereas cues believed to be deviant were closely associated with pretend judgments. Importantly, across both studies, children tended to justify their pretend judgments by referring to absence of real content, despite the fact that we had removed content cues from the test video clips.

\section{General Discussion}

The present work provides evidence of what cues children and adults find most pertinent to judging an act as pretend or real. In this research, 5-year-olds and adults viewed pretense versus real acts, judged whether each act was pretend or real, and justified their judgment by naming the cues on which they based their judgments. The claimed cues suggested what elements of pretense acts are the most pertinent in the viewers' conception of what makes acts be pretense ones.

Adults were very competent in identifying pretense versus real acts even in the absence of content cues. When judging an act as pretense, adults appealed to the characteristics of the act that they believed to be deviant from regular features of the real counterpart. They were most likely to appeal to the nonstandard movements of the character for their pretense interpretations. Adults' attention to the deviant features of the pretense behavior is consistent with the notion that recognition of an activity in the simulative mode often relies on the contrast between the simulation and the counterpart from which it derives [27].

Five-year-old children were relatively competent in identifying pretense versus real acts in the absence of content cues, although their performance was significantly less apt than that of adults. In particular, children in both Studies 1 and 2 falsely claimed that real acts were pretense approximately half the time. Such errors might have resulted from the absence of content cues in the video clips. By 5 years of age, children appear to associate a lack of necessary materials with pretense; when they cannot see whether the real content is there, they guess (50-50) and make their pretend-real judgment based on whatever their guess is. 
In line with this possibility, even 2-year-olds can associate real content with an adult performing a real act and link imaginary or substitute content with a pretending adult $[28,29]$. In the current studies, information about content was eliminated from the video clips. Thus, when the necessary content appears to be "missing," children might take its absence as a cue to pretense. The fact that around $30 \%$ of the time children claimed missing content was the relevant cue when making a pretense interpretation supports this explanation. This is especially interesting given that adults had previewed the test video clips and ascertained that one could not see or hear any direct perceptual information indicating the presence or absence of content in the clips. In this case, then, children's theories about what makes an act a pretense one appear to have trumped the reality of what they could see or hear.

Adults appealing to behavioral markers of pretense and children appealing to missing content suggest that they place differing weights on content versus behavioral cues in their conception of what makes an act a pretense one. For adults, deviant content might be an important marker of pretense, but how a person acts is also clearly an important determinant of whether an act is pretend or real, and when information about content is not available, adults rely on variations in a person's movements. Five-year-olds, however, appear to view deviant content as a more important marker of pretense than behavioral signs. No matter whether or not they can perceive the information about content, if the real content appears to be "missing" in some way, they are likely to judge the act as pretense, paying little attention to the behavioral cues of the person.

These findings might suggest something about how the intuitive theories of pretense held by young children differ from those held by adults. Intuitive theories are lay people's everyday understandings of information in certain domains (e.g., biology, and psychology). These theories have causal powers and serve to guide our interpretations and predictions of events within those domains [30-34]. As many researchers have suggested, intuitive theories imply that children interpret evidence based on their domain-appropriate beliefs and that evidence can help children shape or modify their existing beliefs (e.g., [31,35]). An understanding of pretense is certainly part of adults' intuitive psychological theories; this understanding should be comprised of causal representations of several dimensions, such as why people pretend (and how it relates to other mental states and the world), how people pretend (including its mental origins and physical manifestations), what makes an act appear to be pretense, and what will follow from a pretense premise (psychologically and physically).

The current findings indicate that in children's intuitive theories, an absence of necessary materials could be the most important marker of pretense. Children even claim it is a cue when it is not actually accessible. In contrast, at least when content cues are not available, adults privilege behavioral signs as the right cues to pretense. This does not suggest that deviant content or behavioral signs are actually the defining features of pretense. What defines pretense is the underlying intention to pretend; without such intention, the behavior is not pretense (e.g., $[1,9,36])$. A real act can be intended or unintended, but in either case the underlying causes are different from what leads to pretense acts.

The present findings suggest two future paths for research. First, we examined a specific pretense situation: mothers pretending to have snacks in front of their toddlers. Although this pretense situation is prevalent in many cultures and very frequent in young children's early experience, future work is required to examine whether the present findings can be generalized to pretense situations other than snacking behavior. The second direction for research is what goes along with children moving from a content- to behavior-based interpretation of pretense. It is possible that the shift occurs sometime between 5 and 7 , since by the age of 7 children's judgments are as apt as those of adults even in the absence of content [17].

In conclusion, the present study examines what kinds of evidence children and adults find most pertinent to judging an act as pretend or real. Our findings suggest that, like adults, by 5 years of age children hold intuitive theories of what makes an act a pretense one in the form of contrasts between pretense and its real counterpart. However, in contrast to adults, children appear to place greater weight on deviant content than on behavioral signs to the extent that they even claim to use deviant content when it is imperceptible.

\section{References}

[1] A. S. Lillard, "Pretend play skills and the child's theory of mind," Child development, vol. 64, no. 2, pp. 348-371, 1993.

[2] J. Belsky and R. K. Most, "From exploration to play: a crosssectional study of infant free play behavior," Developmental Psychology, vol. 17, no. 5, pp. 630-639, 1981.

[3] I. Bretherton, "Representing the social world in symbolic play: reality and fantasy," in Symbolic Play: The Development of Social Understanding, I. Bretherton, Ed., pp. 3-41, Academic Press, Orlando, Fla, USA, 1984.

[4] G. G. Fein, "Pretend play in childhood: an integrative review," Child Development, vol. 52, pp. 1095-1118, 1981.

[5] L. Nicolich, "Beyond sensorimotor intelligence: assessment of symbolic maturity through analysis of pretend play," MerrillPalmer Quarterly, vol. 23, no. 2, pp. 89-99, 1977.

[6] W. L. Haight and P. J. Miller, Pretending at Home: Early Development in a Sociocultural Context, SUNY Press, Albany, NY, USA, 1993.

[7] K. L. Walton, Mimesis as Make-Believe, Harvard University Press, Cambridge, Mass, USA, 1990.

[8] A. M. Leslie, "Pretense and representation: the origin of 'theory of mind"' Psychological Review, vol. 94, pp. 412-426, 1987.

[9] A. S. Lillard, "Wanting to be it: children's understanding of intentions underlying pretense," Child Development, vol. 69, no. 4, pp. 981-993, 1998.

[10] H. Rakoczy, M. Tomasello, and T. Striano, "Young children know that trying is not pretending: a test of the "ehaving-as-if" construal of children's early concept of pretense," Developmental Psychology, vol. 40, no. 3, pp. 388-399, 2004.

[11] D. M. Sobel, “Children's knowledge of the relation between intentional action and pretending," Cognitive Development, vol. 22, no. 1, pp. 130-141, 2007. 
[12] A. S. Lillard and D. C. Witherington, "Mothers' behavior modifications during pretense and their possible signal value for toddlers," Developmental Psychology, vol. 40, no. 1, pp. 95113, 2004.

[13] P. H. Weiss, M. Jeannerod, Y. Paulignan, and H. J. Freund, "Is the organisation of goal-directed action modality specific? A common temporal structure," Neuropsychologia, vol. 38, no. 8, pp. 1136-1147, 2000.

[14] M. Bekoff, "Social communication in canids: evidence for the evolution of a stereotyped mammalian display," Science, vol. 197, no. 4308, pp. 1097-1099, 1977.

[15] A. S. Lillard, T. Nishida, D. Massaro, A. Vaish, L. Ma, and G. McRoberts, "Signs of pretense across age and scenario," Infancy, vol. 11, no. 1, pp. 1-30, 2007.

[16] J. S. DeLoache and B. Plaetzer, "Tea for two: joint mother-child symbolic play," in Proceedings of the Biennial Meeting for the Society for Research in Child Development, Toronto, Canada, 1985.

[17] R. A. Richert and A. S. Lillard, "Observers' proficiency at identifying pretense acts based on behavioral cues," Cognitive Development, vol. 19, no. 2, pp. 223-240, 2004.

[18] J. D. Bransford, J. R. Barclay, and J. J. Franks, "Sentence memory: a constructive versus interpretive approach," Cognitive Psychology, vol. 2, pp. 331-350, 1972.

[19] S.-E. Clausen, Applied Correspondence Analysis: An Introduction, Sage, Thousand Oaks, Calif, USA, 1998.

[20] B. S. Everitt, The Analysis of Contingency Tables. Monographs on Statistics and Applied Probability, vol. 4, Chapman \& Hall/CRC, Washington, DC, USA, 1992.

[21] R. Baillargeon, "Object permanence in $31 / 2$ - and 4 1/2-monthold infants," Developmental Psychology, vol. 23, no. 5, pp. 655664, 1987.

[22] E. S. Spelke, K. Breinlinger, J. Macomber, and K. Jacobson, "Origins of knowledge," Psychological Review, vol. 99, no. 4, pp. 605-632, 1992

[23] J. H. Flavell, E. R. Flavell, F. Green, and J. E. Korfmacher, "Do young children think of television images as pictures or real objects," Journal of Broadcasting \& Electronics Media, vol. 34, pp. 399-419, 1990.

[24] A. Dorr, "No shortcuts to judging reality," in Children's Understanding of Television: Research on Attention and Comprehension, J. Bryant and D. R. Anderson, Eds., pp. 199-220, Academic Press, New York, NY, USA, 1983.

[25] C. Hoffner, J. Cantor, and D. M. Badzinski, "Children's understanding of adverbs denoting degree of likelihood," Journal of child language, vol. 17, no. 1, pp. 217-231, 1990.

[26] S. R. Beck, E. J. Robinson, D. J. Carroll, and I. A. Apperly, "Children's thinking about counterfactuals and future hypotheticals as possibilities," Child Development, vol. 77, no. 2, pp. 413-426, 2006.

[27] C. Garvey, Play, Harvard University Press, Cambridge, Mass, USA, 1977.

[28] L. Ma and A. S. Lillard, "Where is the real cheese? Young children's ability to discriminate between real and pretend acts," Child Development, vol. 77, no. 6, pp. 1762-1777, 2006.

[29] L. Ma and A. S. Lillard, "Two-year-olds' understanding of realpretend contrasts: the case of real versus substitute content," in Proceedings of the Biennial Meeting of Society of Research in Child Development, Boston, Mass, USA, 2007.

[30] S. Carey, Conceptual Change in Childhood, MIT Press, Cambridge, Mass, USA, 1985.
[31] A. Gopnik and A. N. Meltzoff, Words, Thoughts, and Theories, MIT Press, Cambridge, Mass, USA, 1997.

[32] K. Inagaki and G. Hatano, "Young children's conception of the biological world," Current Directions in Psychological Science, vol. 15, no. 4, pp. 177-181, 2006.

[33] F. C. Keil, "The origins of an autonomous biology," in Modularity and Constraints in Language and Cognition: The Minnesota Symposia on Child Psychology, M. R. Gunnar and M. Maratsos, Eds., vol. 25, pp. 103-137, Erlbaum, Hillsdale, NJ, USA, 1992.

[34] H. M. Wellman and S. A. Gelman, "Cognitive development: foundational theories of core domains," Annual Review of Psychology, vol. 43, no. 1, pp. 337-375, 1992.

[35] L. E. Schulz, E. B. Bonawitz, and T. L. Griffiths, "Can being scared cause tummy aches? Naive theories, ambiguous evidence, and preschoolers' causal inferences," Developmental Psychology, vol. 43, no. 5, pp. 1124-1139, 2007.

[36] A. K. Hickling, H. M. Wellman, and G. M. Gottfried, "Preschoolers' understanding of others' mental attitudes towards pretend happenings," British Journal of Developmental Psychology, vol. 15, no. 3, pp. 339-354, 1997. 

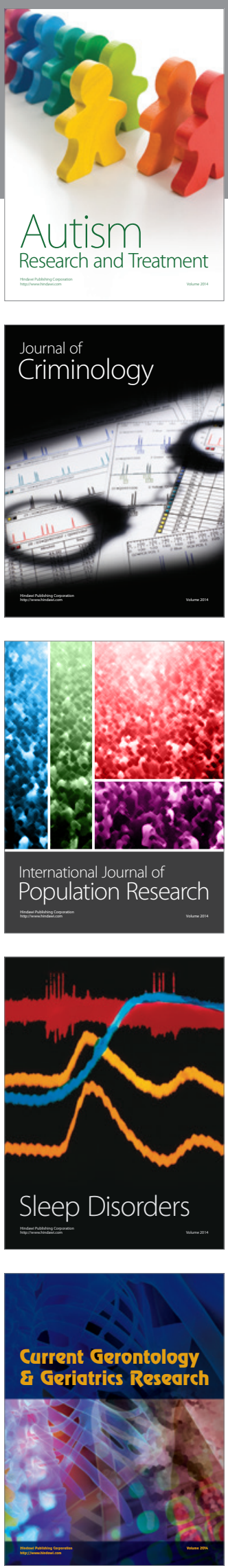
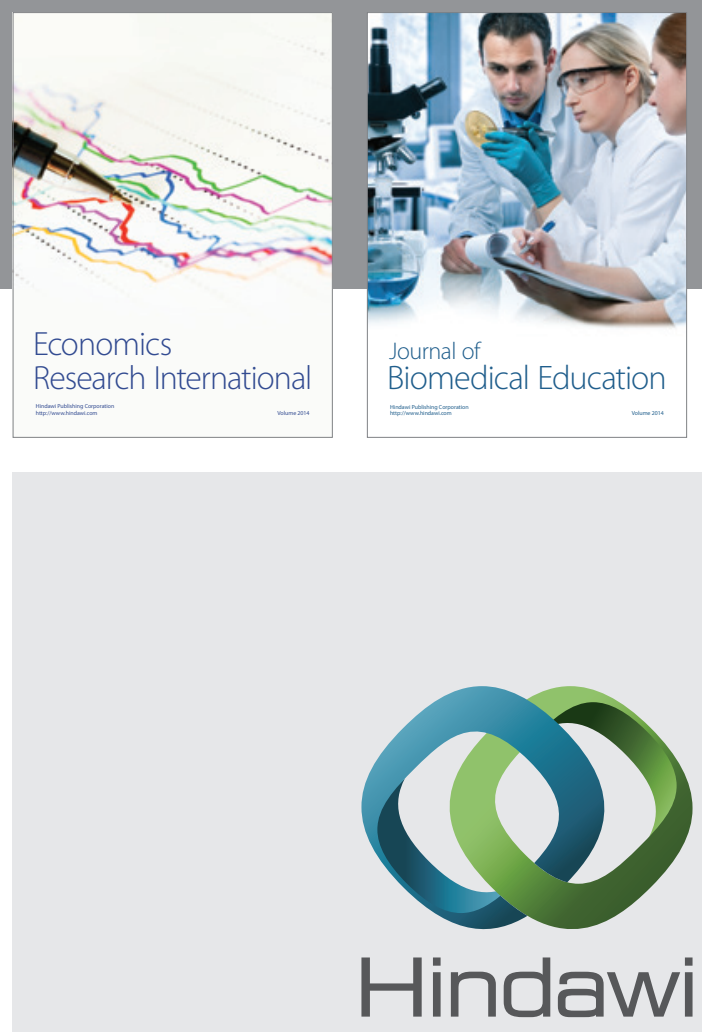

Submit your manuscripts at

http://www.hindawi.com
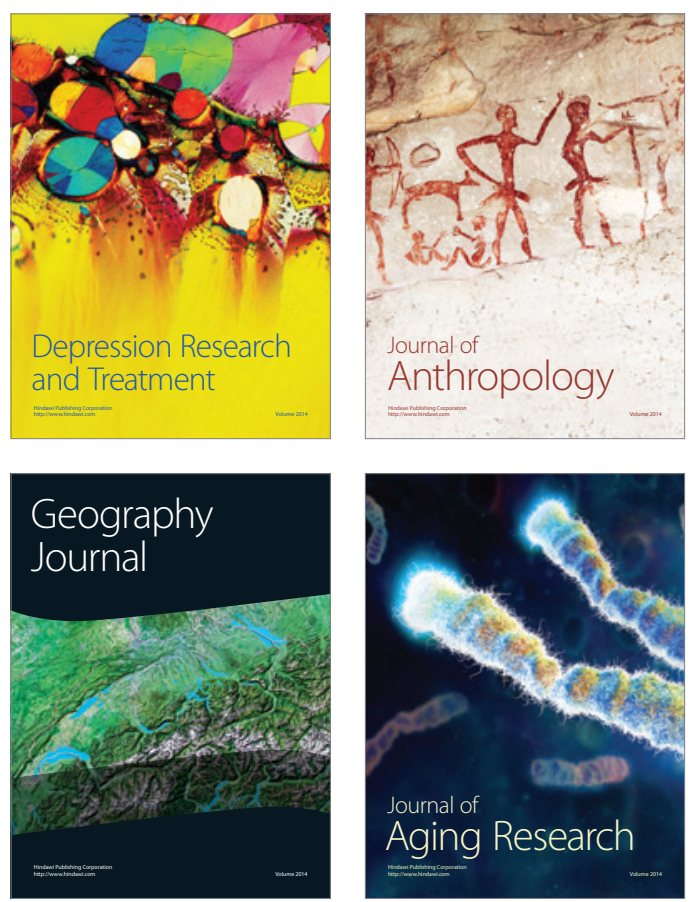
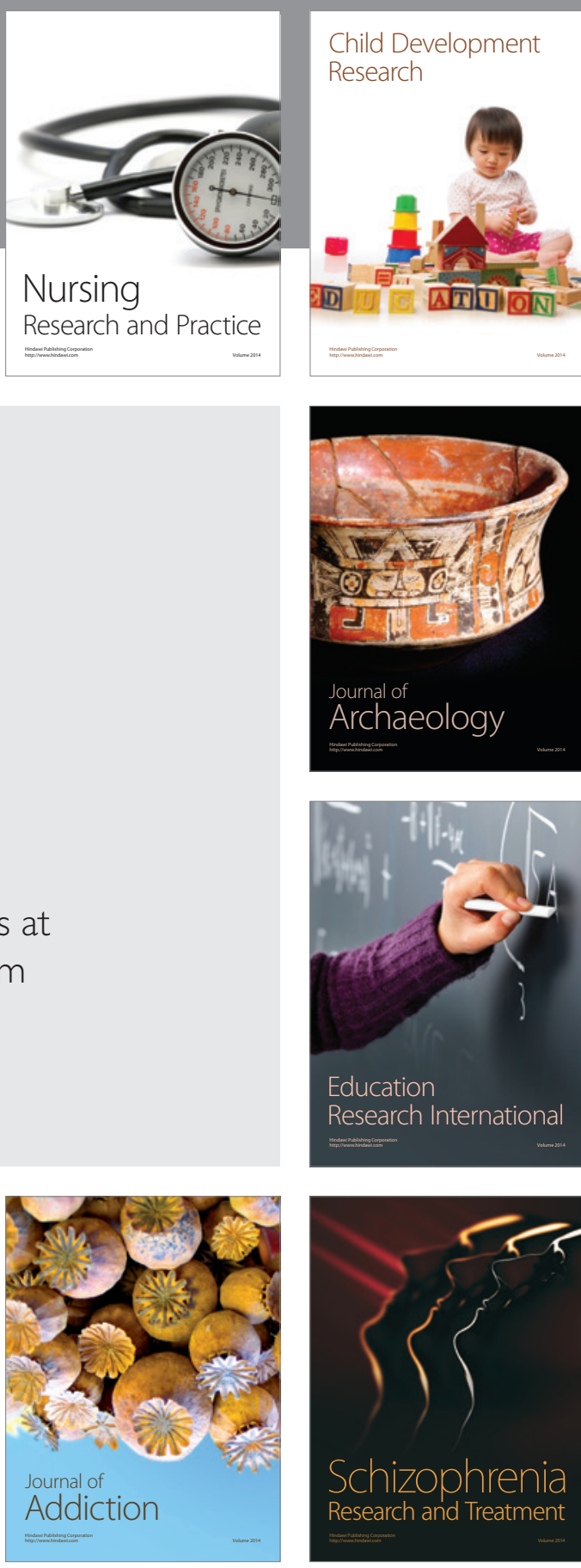

(D)
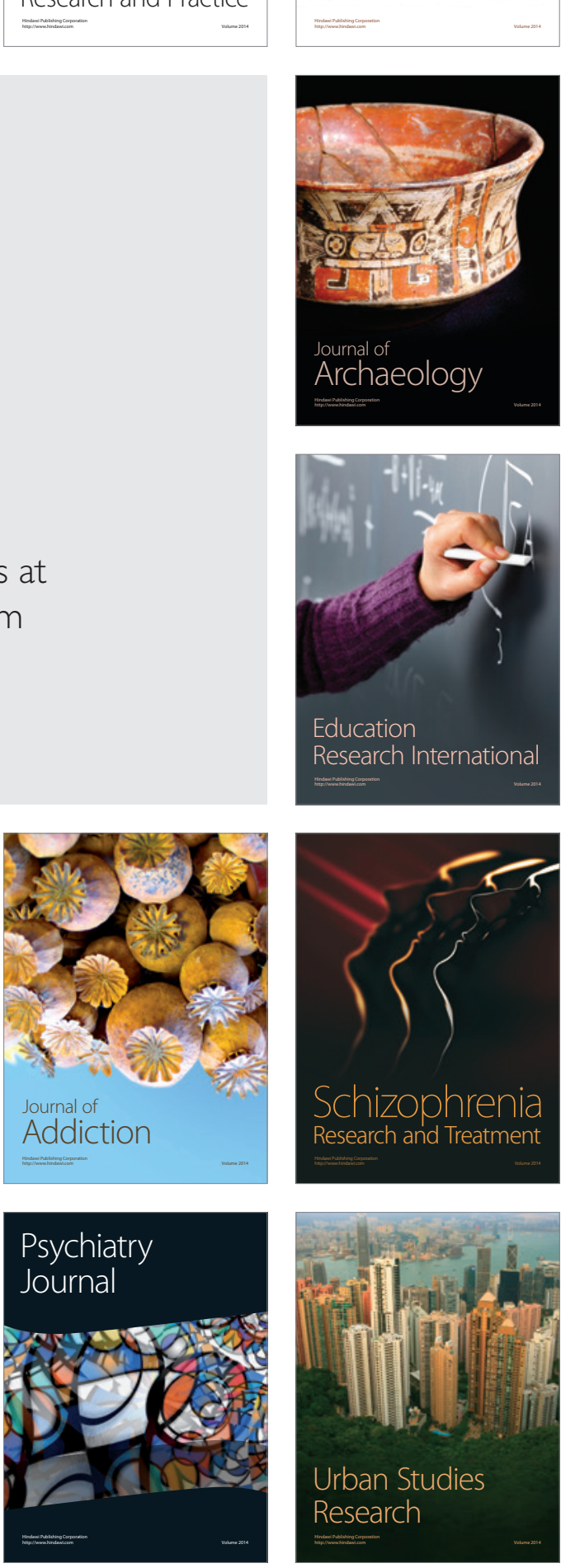